\title{
PROBING DYNAMICAL DARK ENERGY WITH PRESS-SCHECHTER MASS FUNCTIONS
}

\author{
MORGAN LE DELLIOU \\ CFTC, Lisbon University, \\ Lisbon, Portugal \\ delliou@cii.fc.ul.pt
}

\begin{abstract}
This project proposes to discriminate in the wealth of models for dark energy using the formation of non-linear dark matter structures. In particular,it focuses on structures traced by the mass function of dark matter haloes.
\end{abstract}

Keywords: Semi-analytic modeling, Dark matter, Galaxy clusters, Dark energy theory

\section{Introduction}

Among the various models proposed for the explanation of the accelerated expansion in terms of dark energy (DE), distinctions can be made between static (cosmological constant) or dynamical (e.g. quintessence), coupled or uncoupled to dark matter (DM), clustering or unclustering or even unified (Chaplygin Gas) DE. This wealth of models calls for discriminating schemes. The goal of this work is to propose a unified analysis extending previous studies (see Le Delliou 2006, ${ }^{1}$ Manera \& Mota $2006{ }^{2}$ $\mathrm{and}^{3}$ references therein) of DE impact on dark matter haloes on mass functions, for confrontation with other DE assessments.

Table 1. Choice of scalar field's potentials

\begin{tabular}{|c|c|c|c|c|}
\hline \multicolumn{3}{|c|}{ Explored models ${ }^{1,2}$} & \multicolumn{2}{|c|}{ models under scrutiny } \\
\hline Model & Potential V & Origin & Model & Potential V \\
\hline R.P. & $\frac{\Lambda_{Q}^{4+\alpha}}{Q^{\alpha}}$ & $\begin{array}{l}\text { Historical potential; } \\
\text { global SUSY }\end{array}$ & $\begin{array}{c}\text { Albrecht \& } \\
\text { Skordis } 2000^{4}\end{array}$ & $\begin{array}{c}\Lambda_{Q}^{4}\left(\left(\kappa Q-M_{1}\right)^{2}\right. \\
\left.\quad+M_{2}\right) e^{-\lambda \kappa Q}\end{array}$ \\
\hline SUGRA & $\frac{\Lambda_{Q}^{4+\alpha}}{Q^{\alpha}} e^{\kappa^{2} \frac{Q^{2}}{2}}$ & $\begin{array}{c}\text { SUSY+extra } \\
\text { dim.=SUGRA } \\
\text { superpot. }\end{array}$ & $\begin{array}{c}\text { Sahni \& Wang } \\
2000^{5}\end{array}$ & $\begin{array}{c}\Lambda_{Q}^{4}(\cosh (\lambda \kappa Q) \\
-1)^{\alpha}\end{array}$ \\
\hline $\begin{array}{l}\text { Ferreira \& Joyce } \\
1998\end{array}$ & $\Lambda_{Q}^{4} e^{-\lambda \kappa Q}$ & $\begin{array}{c}\text { extra dim. } \\
\text { compactification }\end{array}$ & $\begin{array}{l}\text { Dodelson et al. } \\
\qquad 2000^{6}\end{array}$ & $\begin{array}{c}\Lambda_{Q}^{4} e^{-\lambda \kappa Q}(1+ \\
\alpha \sin (\nu \kappa Q))\end{array}$ \\
\hline $\begin{array}{l}\text { Steinhardt et al. } \\
1999\end{array}$ & $\Lambda_{Q}^{4} e^{\frac{1}{\kappa Q}}$ & $=\sum R \cdot P$ & R.P. $\times$ F.J. & $\frac{\Lambda_{Q}^{4+\alpha}}{Q^{\alpha}} e^{-\lambda \kappa Q}$ \\
\hline $\begin{array}{l}\text { Barreiro et al. } \\
2000\end{array}$ & $\begin{array}{c}\Lambda_{Q}^{4}\left(e^{-\alpha \kappa Q}\right. \\
\left.+e^{-\beta \kappa Q}\right)\end{array}$ & double exponential & $\begin{array}{c}\text { Bertolami et } \\
\text { al. } 2004^{7}\end{array}$ & $\begin{array}{c}V_{0} e^{3(\alpha-1) \phi} \times \\
\left(\left(\cosh \left(\frac{\kappa \phi}{2 / m}\right)\right)^{\frac{2}{\alpha+1}}\right. \\
+\left(\cosh \left(\frac{\kappa \phi}{2 / m}\right)\right)^{\frac{-2 \alpha}{\alpha+1}}\end{array}$ \\
\hline
\end{tabular}

\section{Models and Mass functions}

We model a cosmic fluid with baryons, radiation and, either uncoupled (and coupled) DM with a(n) (un)clustering scalar field DE (quintessence) $Q$, (non)minimally coupled to DM, or a Chaplygin Gas (GCG) - DM/DE unified component - defined either from $P \propto-\rho^{-\alpha}$ or from a scalar field mimicking it. We restrict 
Fig. 1. Cumulative mass functions for different models

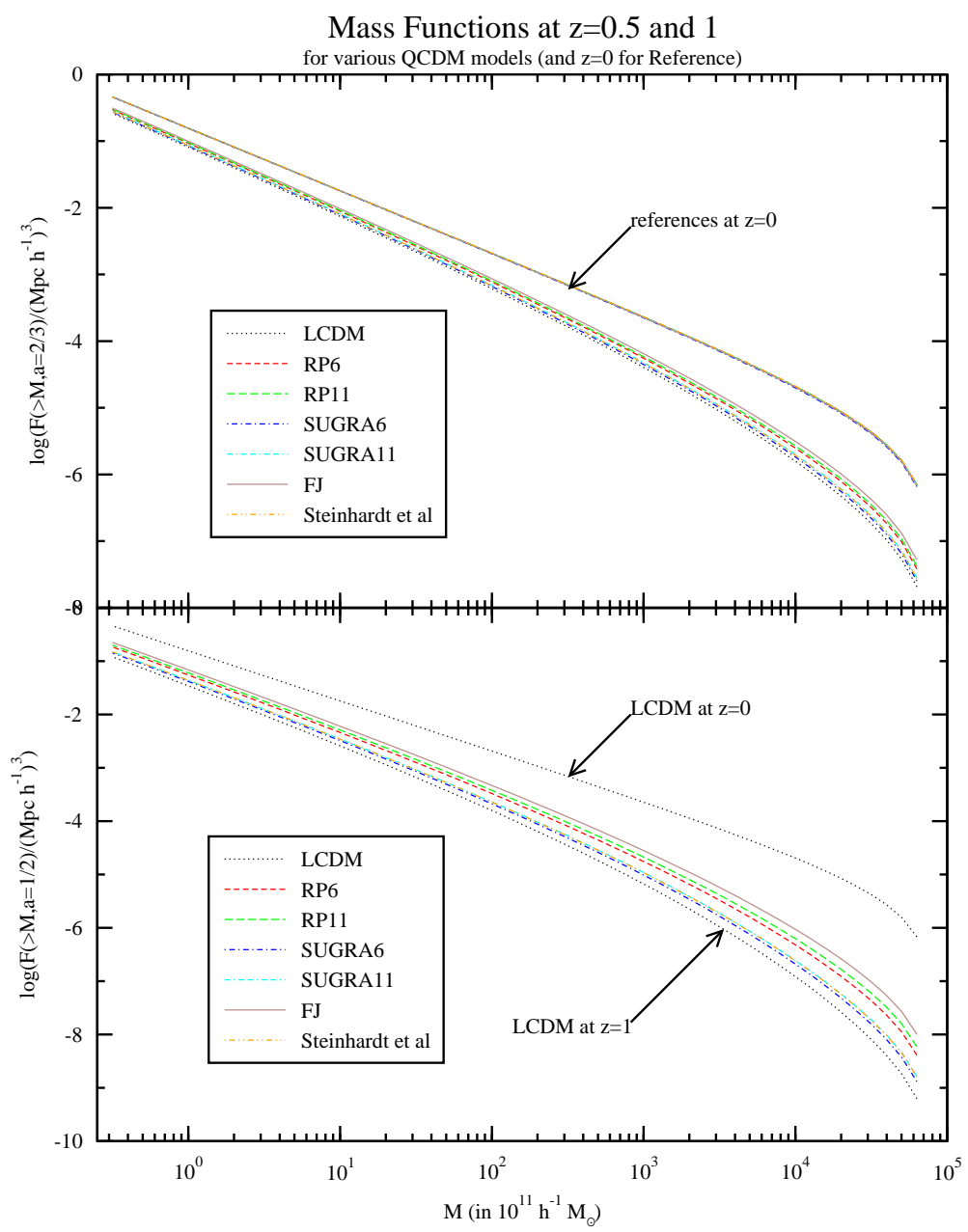

Le Delliou $2006^{1}$
Barreiro et al. model from Manera \& Mota 2006: ${ }^{2}$

$\mathrm{A}=$ non Clust., large $\Omega_{c D M_{0}}$

$\mathrm{B}=$ non Clust., small $\Omega_{c D M_{0}}$

$\mathrm{C}=$ Clust., large $\Omega_{c D M_{0}}$

$\mathrm{D}=$ Clust., small $\Omega_{c D M_{0}}$

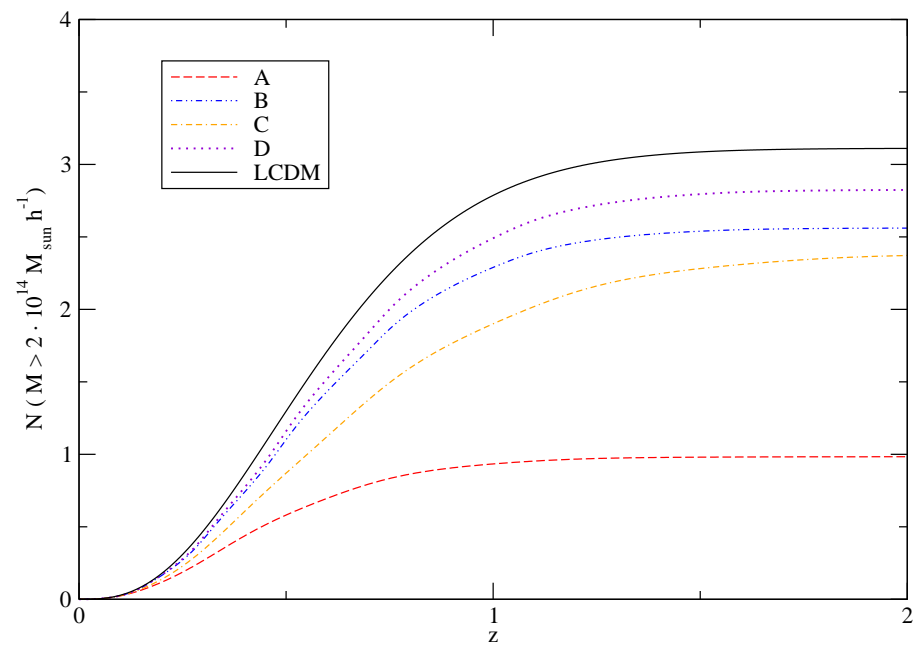

Manera \& Mota $2006^{2}$

to flat backgrounds, a linear coupling and model clustering through energy conservation. The models are defined by their potential (Table 11). We already studied homogeneous minimal quintessences (left side, upper part ${ }^{1}$ ) and a coupled, clustering quintessence (left side, lower part ${ }^{2}$ ). All potentials shown will have clustering and interaction.

We use the top hat spherical collapse to model non-linear structure formation as a Friedmann sphere with higher, varying curvature. We extract the linearly extrapolated overdensity as a function of non-linear collapse scale factor $\delta_{c_{0}}\left(a_{c}\right)$. This is combined in a Press-Schechter scheme to get the mass function of large scale structures. The results obtained so far are presented in Fig. 1. 


\section{Conclusions}

Extending previous evidence of DE models impact on DM mass functions, our results permit the confrontation of several homogeneous models and the examination of clustering and interacting quintessence. This have shown that more insights can be drawn from confrontation of several homogeneous models ${ }^{1}$ and that strong effects on mass function evolution proceed from clustering and interacting quintessence. ${ }^{2}$ Indeed, the spread of $\sim 10 \%$ at $10^{14} h^{-1} M_{\odot}$ between mass functions and the hierarchy between models on the lower $(z=1)$ panel of the left part of Fig. 1 1 shows that the method should be most discriminant on clusters scales and that the impact of $\omega_{Q}$ dominates other effects. Moreover, its right part entails that, contrary to homogeneous models, DE clustering increases DM clustering while coupling decreases it. This motivates our extended study of DE models with mass functions. Some pending questions remain: our use of Birkhoff's theorem with spherical symmetry in cosmology may require some mass function corrections; geometric effects are argued to induce a degeneracy in angular mass functions, ${ }^{8}$ not taking the bias-geometry dependence ${ }^{9}$ into account. We are extending our results to other models (Table 1; Chaplygin gas), including clustering and interacting DE. Further developments are also planned.

\section{Acknowledgements}

See acknowledgements in. ${ }^{1}$ Current work involves J.P. Mimoso, U. Lisboa, D.F. Mota, U. Heidelberg, C. van de Bruck, U. Sheffield and O. Bertolami, IST Lisboa.

\section{References}

1. M. Le Delliou, JCAP 0601, 021 (2006) astro-ph/0506200

2. M. Manera and D. F. Mota, MNRAS 371, 1373 (2006) astro-ph/0504519

3. R. Mainini and S. A. Bonometto, Phys. Rev. D 74, 043504 (2006) astro-ph/0605621

4. A. Albrecht and C. Skordis, Phys. Rev. Lett. 84, 2076 (2000) astro-ph/9908085

5. V. Sahni and L. Wang, Phys. Rev. D 62, 103517 (2000) astro-ph/9910097]

6. S. Dodelson, M. Kaplinghat and E. Stewart, Phys. Rev. Lett 85, 5276 (2000) astro-ph/0002360

7. O. Bertolami, A. A. Sen, S. Sen and P. T. Silva, MNRAS 353, 329 (2004) astro-ph/0402387

8. P. Solevi, R. Mainini and S. A. Bonomento, ApJ (2004) astro-ph/0412054

9. N. Kaiser, ApJ 284, L9 (1984) 